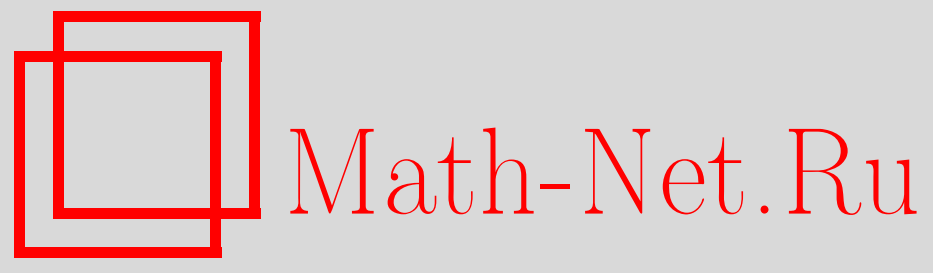

О. А. Репин, С. К. Кумыкова, Об одном классе нелокальных задач для гиперболического уравнения с вырождением типа и порядка, Вестн. Сам. гос. техн. ун-та. Сер. Физ.-мат. науки, 2014, выпуск 4(), 22-32

DOI: https://doi.org/10.14498/vsgtu1348

Использование Общероссийского математического портала MathNet.Ru подразумевает, что вы прочитали и согласны с пользовательским соглашением

http: //www.mathnet.ru/rus/agreement

Параметры загрузки:

IP : 54.209 .52 .79

26 апреля 2023 г., 10:05:53

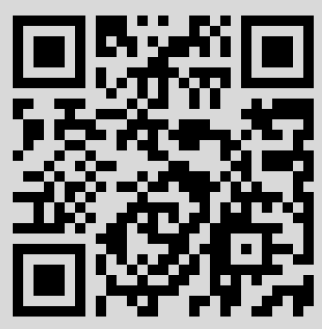


Вестн. Сам. гос. техн. ун-та. Сер. Физ.-мат. науки. 2014. № 4 (37). С. $22-32$

ISSN: 2310-7081 (online), 1991-8615 (print) doi: http://dx.doi.org/10.14498/vsgtu1348

УДК 517.956.326

\title{
ОБ ОДНОМ КЛАССЕ НЕЛОКАЛЬНЫХ ЗАДАЧ ДЛЯ ГИПЕРБОЛИЧЕСКОГО УРАВНЕНИЯ С ВЫРОЖДЕНИЕМ ТИПА И ПОРЯДКА*
}

\author{
О. А. Репин ${ }^{1,2}$, C. К. Кумъкова \\ 1 Самарский государственный экономический университет, \\ Россия, 443090, Самара, ул. Советской Армии, 141. \\ 2 Самарский государственный технический университет, \\ Россия, 443100, Самара, ул. Молодогвардейская, 244. \\ 3 Кабардино-Балкарский государственный университет им. Х. М. Бербекова \\ Россия, 360004, Нальчик, ул. Чернышевского, 173.
}

\section{Аннотация}

В характеристической области исследованы нелокальные задачи для модельного гиперболического уравнения второго порядка, тип и порядок которого вырождается на одной и той же линии $y=0$. C помощью операторов дробного интегро-дифференцирования произвольного порядка на характеристической части границы области задано нелокальное условие, поточечно связывающее дробные производные и интегралы от искомого решения. Для различных значений порядков операторов дробного интегро-дифференцирования, входящих в краевое условие, доказана однозначная разрешимость рассматриваемых задач или установлена неединственность их решения.

Ключевые слова: нелокальная задача, операторы дробного интегро-дифференцирования, задача Коши, интегральное уравнение Вольтерра второго рода, интегральное уравнение Абеля.

doi: http://dx.doi.org/10.14498/vsgtu1348

Введение. Рассмотрим уравнение

$$
y^{2 m} u_{x x}+y u_{y y}+\alpha u_{y}=0,
$$

где $m$ - натуральное число, $\alpha=$ const, $(1-2 m) / 2 \leqslant \alpha<1$ в области $\Omega$, ограниченной характеристиками

$$
A C: x-\frac{2}{2 m+1}(-y)^{\frac{2 m+1}{2}}=0, \quad B C: x+\frac{2}{2 m+1}(-y)^{\frac{2 m+1}{2}}=1
$$

(C) 2014 Самарский государственный технический университет.

\section{Образец для цитирования}

Р епин О. А., Кумыков а С. К. Об одном классе нелокальных задач для гиперболического уравнения с вырождением типа и порядка // Becтн. Сам. гос. техн. ун-та. Сер. Физ.-мат. науки, 2014. № 4 (37). С. 22-32. doi: 10.14498/vsgtu1348.

\section{Сведения об авторах}

Олег Александрович Репин (д.ф.-м.н., проф.; matstat@mail.ru; автор, ведущий переписку), заведующий кафедрой, каф. математической статистики и эконометрики ${ }^{1}$ професcop, каф. прикладной математики и информатики ${ }^{2}$.

Светлана Каншубиевна Кумыкова (к.ф.-м.н., доц.; bsk@rect.kbsu.ru), доцент, каф. теории функций и функционального анализа.

*Настоящая статья представляет собой расширенный вариант доклада [1], сделанного авторами на Четвёртой международной конференции «Математическая физика и её приложения» (Россия, Самара, 25 августа - 1 сентября 2014). 
уравнения (1) и отрезком $I \equiv[0,1]$ прямой $y=0, A(0,0), B(1,0)$.

ЗАДАчА. Найти регулярное в области $\Omega$ решение $u(x, y)$ уравнения (1) из класса $C(\bar{\Omega}) \cap C^{1}(\Omega \cup I)$, удовлетворяющее условиям

$$
\begin{gathered}
u(x, 0)=\tau(x) \quad \forall x \in \bar{I} \\
A(x) D_{0 x}^{a} u\left[\Theta_{0}(x)\right]+B(x) D_{x 1}^{b} u\left[\Theta_{1}(x)\right]=C(x) \quad \forall x \in I .
\end{gathered}
$$

Здесъ $\tau(x), A(x), B(x), C(x)$ - заданные функиии, причём $A^{2}(x)+B^{2}(x) \neq 0$; $\Theta_{0}(x), \Theta_{1}(x)$ - точки пересечения характеристик уравнения $(1)$, выходящих из точки $(x, 0) \in I$, с характеристиками $A C, B C$ соответственно; $D_{0 x}^{\alpha_{1}} f$, $D_{x 1}^{\alpha_{1}} f$-операторы дробного интегрирования порядка $\left(-\alpha_{1}\right)$ при $\alpha_{1}<0 u$ обобщённые производные в смысле Лиувилля порядка $\alpha_{1}>0$ [2, с. 7, 8]; a и b действительные числа, на которые далее будут наложены необходимые условия.

Уравнение (1) служит моделью гиперболических уравнений второго порядка, тип и порядок которых вырождается на одном и том же $(n-1)$-мерном континууме [3, с. 274].

Задача (1)-(3) является нелокальной (задачей со смещением по терминологии А. М. Нахушева [4]). Её исследование связано с прикладным характером задач, возникающих, например, при изучении вопросов тепло- и массообмена в капиллярно-пористых средах.

В монографии [5] широко представлено значение теории дробного интегро-дифференцирования при исследовании нелокальных краевых задач и её проникновение в современную физику.

О возникновении таких задач, их использовании в физике, биологии, математическом моделировании можно узнать, например, в работах [6-8].

Ранее в работе [9] нами была исследована задача с обобщёнными операторами дробного интегро-дифференцирования (в смысле М. Сайго) для вырождающегося гиперболического уравнения. Данная работа является продолжением и обобщением результатов работы [9] для уравнения (1).

В случае

$$
\alpha=1 / 2-m, \quad a=b=1-\beta, \quad \beta=\frac{2 m-1+2 \alpha}{2(2 m+1)}
$$

существование и единственность решения задачи (1)-(3) доказаны А. В. Бицадзе [10].

\section{1. Условия однозначной разрешимости задачи.}

Tеорема 1. Пусть $b=1-\beta, a \leqslant 1-\beta, B(x) \neq 0, \tau(x) \in C^{1}(\bar{I}) \cap C^{3}(I)$, $A(x), B(x), C(x) \in C^{1}(\bar{I})$.

Тогда решение задачи (1)-(3) существует и единственно.

Доказательство. При $(1-2 m) / 2<\alpha<1$ регулярное в области $\Omega$ решение $u(x, y)$ уравнения $(1)$, удовлетворяющее условиям

$$
u(x, 0)=\tau(x), x \in \bar{I}, \quad \lim _{y \rightarrow-0}(-y)^{\alpha} u_{y}=\nu(x), x \in I,
$$

в предположении, что $\tau^{\prime \prime}(x)$ и $\nu^{\prime}(x)$ удовлетворяют условию Гёльдера, единственно и имеет вид [3, с. 277] 


$$
\begin{aligned}
& u(x, y)=\frac{\Gamma(2 \beta)}{\Gamma^{2}(\beta)} \int_{0}^{1} \tau\left[x+\frac{2(1-2 t)}{2 m+1}(-y)^{\frac{2 m+1}{2}}\right][t(1-t)]^{\beta-1} d t- \\
& -\frac{2}{2 m+1} \frac{\Gamma(1-2 \beta)}{\Gamma^{2}(1-\beta)}(-y)^{1-\alpha} \int_{0}^{1} \nu\left[x+\frac{2(1-2 t)}{2 m+1}(-y)^{\frac{2 m+1}{2}}\right][t(1-t)]^{-\beta} d t .
\end{aligned}
$$

Используя формулу (5), находим

$$
\begin{aligned}
& u\left[\Theta_{0}(x)\right]=k_{1} x^{1-2 \beta} D_{0 x}^{-\beta} x^{\beta-1} \tau(x)+k_{2} D_{0 x}^{\beta-1} x^{-\beta} \nu(x), \\
& u\left[\Theta_{1}(x)\right]=k_{1}(1-x)^{1-2 \beta} D_{x 1}^{-\beta}(1-x)^{\beta-1} \tau(x)+k_{2} D_{x 1}^{\beta-1}(1-x)^{-\beta} \nu(x),
\end{aligned}
$$

где

$$
k_{1}=\frac{\Gamma(2 \beta)}{\Gamma(\beta)}, \quad k_{2}=-\frac{\Gamma(1-2 \beta)}{2 \Gamma(1-\beta)}\left(\frac{2 m+1}{4}\right)^{-2 \beta} .
$$

Подставляя (6) в краевое условие (3), учитывая свойства операторов дробного интегро-дифференцирования [11, с. 50, 51]

$$
\begin{gathered}
D_{0 x}^{l} D_{0 x}^{-l} f(x)=D_{x 1}^{l} D_{x 1}^{-l} f(x)=f(x), \\
D_{0 x}^{l} D_{0 x}^{m} f(x)=D_{0 x}^{l+m} f(x), \\
D_{x 1}^{l} D_{x 1}^{m} f(x)=D_{x 1}^{l+m} f(x), \quad l>0, m<0
\end{gathered}
$$

при выполнении условий теоремы 1 , получим

$$
k_{2} B(x)(1-x)^{-\beta} \nu(x)+k_{2} A(x) D_{0 x}^{a+\beta-1} x^{-\beta} \nu(x)=\gamma(x),
$$

где

$$
\begin{aligned}
& \gamma(x)=C(x)-k_{1} A(x) D_{0 x}^{a} x^{1-2 \beta} D_{0 x}^{-\beta} x^{\beta-1} \tau(x)- \\
& \quad-k_{1} B(x) D_{x 1}^{1-\beta}(1-x)^{1-2 \beta} D_{x 1}^{-\beta}(1-x)^{\beta-1} \tau(x) .
\end{aligned}
$$

Или, что то же самое, при $a<1-\beta$

$$
\nu(x)+a_{1}(x) \int_{0}^{x} \frac{t^{-\beta} \nu(t) d t}{(x-t)^{a+\beta}}=f_{1}(x),
$$

где

$$
a_{1}(x)=\frac{A(x)}{B(x)}(1-x)^{\beta}, \quad f_{1}(x)=\frac{\gamma(x)}{k_{2} B(x)}(1-x)^{\beta} .
$$

Исследуем правую часть $f_{1}(x)$ уравнения (8).

Воспользуемся формулой композиции для операторов дробного интегродифференцирования, доказанной в [12],

$$
I_{1}(x)=D_{x 1}^{1-\beta}(1-x)^{1-2 \beta} D_{x 1}^{-\beta}(1-x)^{\beta-1} \tau(x)=(1-x)^{-\beta} D_{x 1}^{1-2 \beta} \tau(x) .
$$

Рассмотрим два случая изменения параметра $a$ :

1) $a \leqslant 0$,

2) $0<a \leqslant 1-\beta$. 
Исследуем

$$
I_{2}(x)=D_{0 x}^{a} x^{1-2 \beta} D_{0 x}^{-\beta} x^{\beta-1} \tau(x) .
$$

В результате ряда преобразований (11) примет вид

$$
I_{2}(x)=\frac{\Gamma(\beta-a) x^{-a}}{\Gamma^{2}(\beta) \Gamma^{2}(-a)} \int_{0}^{1} \frac{(1-z)^{\beta-1-a} \tau(x z)}{z^{1-\beta}} F(2 \beta-1,-a ; \beta-a ; 1-z) d z .
$$

При $a<0$ в силу (9), (10), (11), (12) и условий теоремы $1(1-x)^{\beta} I_{1}(x) \in$ $C[0,1)$, а при $x=1$ может обращаться в бесконечность порядка $1-2 \beta$; $(1-x)^{\beta} I_{2}(x) \in C(\bar{I})$, а при $x=0$ и $x=1$ обращается в ноль.

При $a=0$

$$
(1-x)^{\beta} I_{2}(x)=\frac{(1-x)^{\beta}}{\Gamma(\beta)} \int_{0}^{1} \frac{\tau(x z) d z}{[z(1-z)]^{1-\beta}} .
$$

Следовательно, $(1-x)^{\beta} I_{2}(x) \in C(\bar{I})$.

Рассмотрим случай, когда $0<a \leqslant 1-\beta$. Так как параметр $a$ в $I_{1}(x)$ отсутствует, исследуем только $I_{2}^{*}(x)=(1-x)^{\beta} I_{2}(x)$ :

$$
I_{2}^{*}(x)=\frac{(1-x)^{\beta}}{\Gamma(\beta) \Gamma(1-a)} \frac{d}{d x} \int_{0}^{x} \frac{t^{1-\beta} d t}{(x-t)^{a}} \int_{0}^{t} \frac{\xi^{\beta-1} \tau(\xi) d \xi}{(t-\xi)^{1-\beta}} .
$$

Поменяв порядок интегрирования, а затем продифференцировав, будем иметь

$$
\begin{aligned}
& I_{2}^{*}(x)=\frac{(1-x)^{\beta}}{\Gamma(1-a+\beta)} \int_{0}^{1} \frac{(1-a) x^{-a} \tau(x z)+x^{1-a} z \tau^{\prime}(x z)}{z^{1-\beta}(1-z)^{a-\beta}} \times \\
& \times F(2 \beta-1,1-a ; 1-a+\beta ; 1-z) d z .
\end{aligned}
$$

Поскольку $a \leqslant 1-\beta$, справедливы неравенства $a-\beta \leqslant 1-2 \beta, 0<1-$ $-2 \beta<1$. Следовательно, $I_{2}^{*}(x) \in C(0,1]$ и при $x=0$ может обращаться в бесконечность порядка $a$.

Таким образом, при выполнении условий теоремы 1 вопрос существования решения задачи (1)-(3) редуцирован к вопросу разрешимости интегрального уравнения Вольтерра второго рода со слабой особенностью в ядре

$$
\nu(x)+\int_{0}^{x} \frac{a_{1}(x) \nu(t) d t}{t^{\beta}(x-t)^{a+\beta}}=f_{1}(x),
$$

где $f_{1}(x) \in C^{1}(I)$ и при $x=0$ может обращаться в бесконечность порядка $a$, если $0<a<1-\beta$, а при $x=1$ может обращаться в бесконечность порядка $1-2 \beta$. Единственное решение уравнения (13) может быть построено методом последовательных приближений.

ЗАмЕчАниЕ. Если $a=1-\beta$, то из (7) сразу находим

$$
\nu(x)=\frac{\gamma(x)}{k_{2}\left[(1-x)^{-\beta} B(x)+x^{-\beta} A(x)\right]}
$$

и решение $u(x, y)$ записываем по формуле $(5)$. 


\section{2. Случаи неединственности решения задачи.}

Теорема 2. Пусть выполнены условия $b=1-\beta, 1-\beta<a \leqslant 2-\beta$; $\nu(x)=x^{a+2 \beta-2} \nu_{1}(x), \nu_{1}(0) \neq 0, \nu_{1}(x) \in C^{1}(\bar{I}) ; \tau(x)=x^{\sigma} \tau_{1}(x), \sigma \geqslant a$, $\tau_{1}(x) \in C^{2}(\bar{I}) \cap C^{4}(I) ; B(x)=(1-x)^{\beta} b_{2}(x) ; A(x), b_{2}(x) \in C(\bar{I}) \cap C^{1}(I)$; $A(x) \cdot b_{2}(x) \neq 0 \forall x \in \bar{I}$.

Тогда задача (1)-(3) имеет более одного решения.

Доказ ат ельств о. Рассмотрим случай, когда $b=1-\beta, a=2-\beta$.

Из (7) при $A(x) \neq 0$ имеем

$$
\nu^{\prime}(x)+\left[\frac{B(x)}{A(x)}\left(\frac{x}{1-x}\right)^{\beta}-\frac{\beta}{x}\right] \nu(x)=\frac{x^{\beta} \gamma(x)}{k_{2} A(x)} .
$$

Уравнение (14) является линейным обыкновенным дифференциальным уравнением первого порядка и решение его, содержащее произвольную постоянную, можно выписать согласно общей теории. В силу этого решение задачи (1)-(3) не единственно.

Теперь рассмотрим случай, когда $b=1-\beta, 1-\beta<a<2-\beta, A(x) \neq 0$.

С учетом условий теоремы 2 уравнение (7) принимает вид

$$
b_{2}(x) \nu(x)+a_{2}(x) \frac{d}{d x} \int_{0}^{x} \frac{t^{-\beta} \nu(t) d t}{(x-t)^{a+\beta-1}}=\frac{1}{k_{2}} \gamma(x),
$$

где

$$
a_{2}(x)=\frac{A(x)}{\Gamma(2-a-\beta)} .
$$

Исследуем гладкость $\gamma(x)$ - правой части уравнения (15).

Нетрудно показать, что

$$
\begin{aligned}
& I_{1}(x)=(1-x)^{-\beta} D_{x 1}^{1-2 \beta} \tau(x)= \\
& \quad=(1-x)^{-\beta}\left[\frac{\tau(1)}{\Gamma(2 \beta)(1-x)^{1-2 \beta}}-\frac{1}{\Gamma(2 \beta)} \int_{x}^{1} \frac{\tau^{\prime}(t) d t}{(t-x)^{1-2 \beta}}\right] ; \\
& \widetilde{I}_{2}(x)=D_{0 x}^{a} x^{1-2 \beta} D_{0 x}^{-\beta} x^{\beta-1} \tau(x)=\frac{d^{2}}{d x^{2}} D_{0 x}^{a-2} x^{1-2 \beta} D_{0 x}^{-\beta} x^{\beta-1} \tau(x)= \\
& =\frac{1}{\Gamma(2-a+\beta)} \frac{d^{2}}{d x^{2}} x^{2-a} \int_{0}^{1} \frac{\tau(x z)}{z^{1-\beta}(1-z)^{a-\beta+1}} F(2 \beta-1,2-a ; 2-a+\beta ; 1-z) d z .
\end{aligned}
$$

Дифференцируя дважды $\widetilde{I}_{2}(x)$, в результате несложных преобразований с учетом условий теоремы 2 можно заключить, что $I_{1}(x) \in C[0,1) \cap C^{1}(I)$ и при $x=1$ может обращаться в бесконечность порядка $1-\beta, \widetilde{I}_{2}(x) \in C(\bar{I}) \cap$ $C^{(1, \mu)}(I), 0<\mu \leqslant 1$.

Итак, правая часть уравнения $(15) \gamma(x) \in C[0,1) \cap C^{1}(I)$ и при $x=1$ может обращаться в бесконечность порядка $1-\beta$. 
Для доказательства теоремы 2 достаточно показать, что однородное уравнение, соответствующее (15),

$$
b_{2}(x) \nu(x)+a_{2}(x) \frac{d}{d x} \int_{0}^{x} \frac{t^{-\beta} \nu(t) d t}{(x-t)^{a+\beta-1}}=0
$$

имеет нетривиальное решение.

Аналогично [9] введем новую неизвестную функцию

$$
\varphi(x)=\int_{0}^{x} \frac{t^{-\beta} \nu(t) d t}{(x-t)^{a+\beta-1}}
$$

и применим формулу обращения

$$
f(x)=\frac{\sin (\pi \mu)}{\pi} \frac{d}{d x} \int_{0}^{x} \frac{F(t) d t}{(x-t)^{1-\mu}}
$$

интегрального уравнения Абеля [11, с. 38, 39]

$$
\int_{0}^{x} \frac{f(t) d t}{(x-t)^{\mu}}=F(x), \quad 0<\mu<1
$$

к уравнению (17).

В результате получим

$$
\nu(x)=\frac{x^{\beta} \sin (\pi[a+\beta-1])}{\pi} \frac{d}{d x} \int_{0}^{x} \frac{\varphi(t) d t}{(x-t)^{2-a-\beta}} .
$$

Подставляя $\nu(x)$ в $(16)$, после преобразований будем иметь

$$
\begin{aligned}
a_{2}(x) \frac{d}{d x} \varphi(x) & +\frac{x^{\beta-1} b_{2}(x) \sin (\pi[a+\beta-1])}{\pi} \times \\
& \times\left[(a+\beta-1) \int_{0}^{x} \frac{\varphi(z) d z}{(x-z)^{2-a-\beta}}+\int_{0}^{x} \frac{z \varphi^{\prime}(z) d z}{(x-z)^{2-a-\beta}}\right]=0 .
\end{aligned}
$$

Из (17) с учетом условий теоремы 2 следует

$$
\varphi(x)=\int_{0}^{1} z^{a+\beta-2}(1-z)^{1-\beta-a} \nu_{1}(x z) d z
$$

и

$$
\varphi(0)=B(a+\beta-1,2-\beta-a) \nu_{1}(0)=c_{0}=\text { const } \neq 0 .
$$

Обозначив

$$
\psi(x)=\frac{d}{d x} \varphi(x)
$$

будем иметь

$$
\varphi(x)=c_{0}+\int_{0}^{x} \psi(t) d t .
$$


Подставляя (20) и (21) в (19), после некоторых преобразований получим

$$
\psi(x)+a^{*}(x) \int_{0}^{x} \frac{\psi(t) d t}{(x-t)^{2-a-\beta}}=x^{a+2 \beta-2} h(x),
$$

где

$$
a^{*}(x)=\frac{\sin (\pi[a+\beta-1]) x^{\beta} b_{2}(x)}{\pi a_{2}(x)}, \quad h(x)=-\frac{c_{0} \sin (\pi[a+\beta-1]) x^{a+2 \beta-2} b_{2}(x)}{\pi a_{2}(x)} .
$$

Уравнение (22) - уравнение Вольтерра второго рода. Методом последовательных приближений можно показать, что оно имеет решение в классе функций $\psi(x)=x^{a+2 \beta-2} \psi_{1}(x)$, где $\psi_{1}(x) \in C(\bar{I}) \cap C^{2}(I)$. Следовательно, существует нетривиальное решение уравнения (22) и делается заключении о неединственности решения задачи (1)-(3).

Докажем существование решения задачи.

Уравнение (15), с учетом замен (17) и (20), примет вид

$$
\psi(x)+\int_{0}^{x} \frac{k(x, a) \psi(t) d t}{(x-t)^{2-a-\beta}}=f_{2}(x)
$$

где

$$
k(x, a)=a^{*}(x), \quad f_{2}(x)=h(x) x^{a+2 \beta-2}+\frac{\gamma(x)}{k_{2} a_{2}(x)} .
$$

Учитывая условия теоремы 2 и проведенные вычисления, заметим, что $f_{2}(x)$ представимо в виде

$$
f_{2}(x)=x^{a+2 \beta-2}(1-x)^{\beta-1} \overline{f_{2}}(x),
$$

где $\overline{f_{2}}(x) \in C(\bar{I})$.

Уравнение (23) - уравнение Вольтерра второго рода. Оно имеет нетривиальное решение в классе функций, к которому принадлежит правая часть $f_{2}(x)$.

По найденному $\psi(x)$ определяется $\varphi(x)$ из $(21)$ и $\nu(x)$ из $(18)$, а следовательно, и решение задачи (1))-(3) по формуле (5).

Далее регулярным решением уравнения (1) в области $\Omega$ назовем функцию $u(x, y) \in C(\bar{I}) \cap C^{2}(\Omega)$, удовлетворяющую уравнению (1) и такую, что $u_{y}(x, 0)=x^{a+2 \beta-2} \nu_{1}(x)$, а $\nu_{1}(x)$ - достаточное число раз дифференцируемая функция в некоторой окрестности $(0, \delta)$ точки $x=0$ и $\nu_{1}(0) \neq 0$.

Teоpema 3. Пусть $b=1-\beta, 1-\beta+k<a<2-\beta+k, k=1,2, \ldots$; $\tau(x)=x^{\sigma} \tau_{1}(x), \sigma \geqslant a, \tau_{1}(x) \in C^{k+1}(\bar{I}) ; B(x)=x(1-x)^{1-\beta} b_{3}(x)$, $A(x), b_{3}(x) \in C(\bar{I}), A(x) b_{3}(x) \neq 0 \forall x \in \bar{I}$.

Тогда задача (1)-(3) имеет бесчисленное множество линейно независимых регулярных решений.

Д о каз ат ель с т в о. Покажем, что теорема 3 справедлива при $k=1$. В этом случае уравнение (7) примет вид

$$
x(1-x)^{1-2 \beta} b_{3}(x)+a_{3}(x) \frac{d^{2}}{d x^{2}} \int_{0}^{x} \frac{t^{-\beta} \nu(t) d t}{(x-t)^{a+\beta-2}}=\gamma(x),
$$


где

$$
a_{3}(x)=\Gamma(3-a-\beta) A(x) .
$$

Для доказательства теоремы 3 достаточно показать, что соответствующее (24) однородное уравнение имеет нетривиальное решение.

Как и ранее, обозначая

$$
\varphi(x)=\int_{0}^{x} \frac{t^{-\beta} \nu(t) d t}{(x-t)^{a+\beta-2}}
$$

и применяя формулу обращения интегрального уравнения Абеля, получим

$$
\begin{aligned}
a_{3}(x) \frac{d^{2}}{d x^{2}} \varphi(x) & +x^{\beta}(1-x)^{1-2 \beta} \frac{\sin (\pi[a+\beta-2])}{\pi} b_{3}(x) \times \\
& \times\left[(a+\beta-2) \int_{0}^{x} \frac{\varphi(t) d t}{(x-t)^{3-a-\beta}}+\int_{0}^{x} \frac{t \varphi^{\prime}(t) d t}{(x-t)^{3-a-\beta}}\right]=0 .
\end{aligned}
$$

Из (25) легко видеть, что

$$
\varphi(0)=0, \quad \varphi^{\prime}(0)=B(a+\beta-1,3-a-\beta) \nu_{1}(0)=c_{1} \neq 0 .
$$

Пологая

$$
\frac{d^{2}}{d x^{2}} \varphi(x)=\psi(x)
$$

и интегрируя дважды, будем иметь

$$
\varphi(x)=\int_{0}^{x}(x-\xi) \psi(\xi) d \xi+c_{1} x, \quad c_{1}=\text { const. }
$$

Пусть $a_{3}(x) \neq 0$. Учитывая $(27)$, уравнению $(26)$ можно придать вид

$$
\psi(x)+\int_{0}^{x} \psi(\xi) K_{1}(x, \xi) d \xi=\gamma_{1}(x)
$$

где

$$
\begin{gathered}
K_{1}(x, \xi)=\frac{\sin (\pi[a+\beta-2])}{\pi(a+\beta-2)} \frac{b_{3}(x)}{a_{3}(x)} x^{1+\beta}(1-x)^{1-2 \beta}, \\
\gamma_{1}(x)=-\frac{\sin (\pi[a+\beta-2])}{\pi} \frac{b_{3}(x)}{a_{3}(x)} x^{a+2 \beta-1}(1-x)^{1-2 \beta} .
\end{gathered}
$$

Так как $2-\beta-a<0$, уравнение (28) является интегральным уравнением Вольтерра второго рода с ядром $K_{1}(x, \xi) \in C(\bar{I} \times \bar{I})$ и непрерывной правой частью $\gamma_{1}(x)$.

Известно [13], что уравнение (28) имеет на $\bar{I}$ единственное непрерывное решение, которое определяется по формуле

$$
\psi(x)=\gamma_{1}(x)+\int_{0}^{x} R(x, t) \gamma_{1}(t) d t
$$

где $R(x, t)$ - резольвента ядра $K_{1}(x, t)$. 
Следовательно, при $k=1$ решение задачи (1)-(3) не единственно.

Для доказательства существования решения задачи вернемся к уравнению (24) и, проделывая те же вычисления, получим уравнение Вольтерра второго рода

$$
\psi(x)+\int_{0}^{x} \psi(\xi) K_{1}(x, \xi) d \xi=\gamma_{1}(x)+\frac{\gamma(x)}{a_{3}(x)}
$$

с непрерывным ядром и непрерывной правой частью, которое имеет единственное непрерывное решение в классе непрерывных функций.

Применяя метод математической индукции, аналогично [9], можно доказать теорему 3 , если $k-1<a+\beta-1<k$.

Принадлежность $\nu(x)$ классу $C^{1}(I)$ обеспечивается гладкостью известных функций.

Нетрудно доказать справедливость следующего утверждения.

Tеорема 4. Если $a=n+1-\beta, b=1-\beta, n=1,2, \ldots ; B(x)=(1-x)^{1-\beta} b(x)$, $A(x), b(x), \gamma(x) \in C(\bar{I}), A(x) \neq 0 \forall x \in \bar{I} ; \tau(x)=x^{\sigma} \tau_{1}(x), \sigma \geqslant n+1-\beta$, $\tau_{1}(x) \in C^{n+1}(\bar{I})$, где $n-$ иелая часть $a$, то при $\nu_{1}(0) \neq 0$ задача $(1)-(3)$ имеет более одного регулярного решения.

Таким образом, установлены промежутки изменения порядков операторов дробного интегро-дифференцирования в смысле Римана-Лиувилля, входящих в краевое условие и связанных с параметрами рассматриваемого уравнения, при которых исследуемые задачи либо однозначно разрешимы, либо доказана неединственность их решений.

\section{ORCID}

Олег Александрович Репин: http://orcid.org/0000-0003-1522-3955

\section{БИБЛИОГРАФИЧЕСКИЙ СПИСОК}

1. Репин О. А., Кумыкова С. К. Об одном классе нелокальных задач для гиперболического уравнения с вырождением типа и порядка/ Четвертая международная конферениия «Математическая физика и ее приложения»: материалы конф.; ред. чл.корр. РАН И. В. Волович; д.ф.-м.н., проф. В. П. Радченко. Самара: СамГТУ, 2014. C. 299 .

2. Нахушев А. М. Дробное исчисление и его применение. М.: Физматлит, 2003. 272 с.

3. Бицадзе А. В. Некоторые классы уравнений в частных производных. М.: Наука, 1981. 448 c.

4. Нахушев А. М. О некоторых новых краевых задачах для гиперболических уравнений и уравнений смешанного типа // Диффер. уравн., 1969. Т. 5, № 1. С. 44-59.

5. Учайкин В. В. Метод дробных производнъх. Ульяновск: Артишок, 2008. 512 с.

6. Mainardi F. Fractional Calculus / Fractals and Fractional Calculus in Continuum Mechanics/ International Centre for Mechanical Sciences, 378; eds. A. Carpinteri, F. Mainardi. Wien: Springer, 1997. pp. 291-348. doi : 10.1007/978-3-7091-2664-6_7.

7. Nigmatulin R. R. The realization of generalized transfer equation in a medium with fractal geometry // Physica Status Solidi (B), 1986. vol.133, no.1. pp. 425-430. doi: 10.1002/ pssb. 2221330150.

8. Saichev A. I., Zaslavsky G. M. Fractional kinetic equations: solutions and applications // Chaos, 1997. vol. 7, no. 4. pp. 753-764. doi: 10.1063/1.166272.

9. Репин О. А., Кумыкова С. К. Задача с обобщенными операторами дробного интегродифференцирования произвольного порядка // Изв. вузов. Матем., 2012. №12. С. 5971. 
10. Бицадзе А. В. К теории уравнений смешанного типа, порядок которых вырождается вдоль линии изменения типа/ Механика сплошной среды и родственные проблемы анализа: сб. тр., посвящ. 80-летию Н. И. Мусхелишвили. М.: Наука, 1972. С. 48-52.

11. Самко С. Г., Килбас А. А., Маричев О. И. Интеграль и производные дробного порядка и некоторые их приложения. Минск: Наука и техника, 1987. 688 с.

12. Кумыкова С. К. Об одной задаче с нелокальными краевыми условиями на характеристиках для уравнения смешанного типа // Диффер. уравн., 1974. Т. 10, № 1. С. 78-88.

13. Трикоми Ф. Интегральные уравнения. М.: Иностр. литер., 1960. 299 с.

Поступила в редакцию $23 / \mathrm{X} / 2014$;

в окончательном варианте - 05/XI/2014;

принята в печать $-27 / \mathrm{XI} / 2014$.

Vestn. Samar. Gos. Techn. Un-ta. Ser. Fiz.-mat. nauki

[J. Samara State Tech. Univ., Ser. Phys. \& Math. Sci.] 2014. Issue 4 (37). Pp. 22-32

ISSN: 2310-7081 (online), 1991-8615 (print)

doi: http://dx.doi.org/10.14498/vsgtu1348

MSC: 35M12

\title{
ON A CLASS OF NONLOCAL PROBLEMS FOR HYPERBOLIC EQUATIONS WITH DEGENERATION OF TYPE AND ORDER*
}

\author{
O. A. Repin ${ }^{1,2}$, S. K. Kumykova ${ }^{3}$ \\ 1 Samara State Economic University, \\ 141, Sovetskoy Armii st., Samara, 443090, Russian Federation. \\ 2 Samara State Technical University, \\ 244, Molodogvardeyskaya st., Samara, 443100, Russian Federation. \\ 3 Kabardino-Balkarian State University, \\ 173, Chernyshevskogo st., Nalchik, 360004, Russian Federation.
}

\begin{abstract}
Nonlocal problems for the second order hyperbolic model equation were studied in the characteristic area. The type and order of equations degenerate on the same line $y=0$. Nonlocal condition is given by means of fractional integro-differentiation of arbitrary order on the boundary. Nonlocal condition connects fractional derivatives and integrals of the desired solution. For different values of order operators of fractional integro-differentiation within the boundary condition the unique solvability of the considered problems was proved or non-uniqueness of the solution was estimated.
\end{abstract}

(C) 2014 Samara State Technical University.

How to cite Reference

Repin O. A., Kumykova S. K. On a class of nonlocal problems for hyperbolic equations with degeneration of type and order, Vestn. Samar. Gos. Tekhn. Univ., Ser. Fiz.-Mat. Nauki [J. Samara State Tech. Univ., Ser. Phys. \& Math. Sci.], 2014, no. 4(37), pp. 22-32. doi: 10.14498/vsgtu1348. (In Russian)

Authors Details

Oleg A. Repin (Dr. Phys. \& Math. Sci., Professor; matstat@mail.ru; Corresponding Author), Head of Department, Dept. of Mathematical Statistics and Econometrics ${ }^{1}$; Professor, Dept. of Applied Mathematics \& Computer Science ${ }^{2}$. Svetlana K. Kumykova (Cand. Phys. \& Math. Sci.; bsk@rect.kbsu.ru), Associate Professor, Dept. of Function Theory.

${ }^{*}$ This paper is an extended version of the paper [1], presented at the Mathematical Physics and Its Applications 2014 Conference. 
Keywords: nonlocal boundary value problem, fractional integro-differentiation operators, Cauchy problem, second kind Volterra integral equation, Abel integral equation.

doi: http://dx.doi.org/10.14498/vsgtu1348

\section{ORCID}

Oleg A. Repin: http://orcid.org/0000-0003-1522-3955

\section{REFERENCES}

1. Repin O. A., Kumykova S. K. On a class of nonlocal problems for hyperbolic equations with degeneration of type and order, The 4nd International Conference "Mathematical Physics and its Applications", Book of Abstracts and Conference Materials; eds. I. V. Volovich; V. P. Radchenko. Samara, Samara State Technical Univ., 2014, pp. 299 (In Russian).

2. Nakhushev A. M. Drobnoe ischislenie $i$ ego primenenie [Fractional calculus and its applications]. Moscow, Fizmatlit, 2003, 272 pp. (In Russian)

3. Bitsadze A. V. Some classes of partial differential equations, Advanced Studies in Contemporary Mathematics, vol.4. New York, Gordon \& Breach Science Publ., 1988, $\mathrm{xi}+504 \mathrm{pp}$.

4. Nakhushev A. M. Certain boundary-value problems for hyperbolic equations and for equations of mixed type, Differ. Equ., 1969, vol. 5, no. 1, pp. 37-57.

5. Uchaikin V. V. Metod drobnykh proizvodnykh [Method of Fractional Derivatives]. Ul'ianovsk, Artishok, 2008, 512 pp. (In Russian)

6. Mainardi F. Fractional Calculus, Fractals and Fractional Calculus in Continuum Mechanics, International Centre for Mechanical Sciences, 378; eds. A. Carpinteri, F. Mainardi. Wien, Springer, 1997, pp. 291-348. doi: 10.1007/978-3-7091-2664-6_7.

7. Nigmatulin R. R. The realization of generalized transfer equation in a medium with fractal geometry, Physica Status Solidi (B), 1986, vol. 133, no. 1, pp. 425-430. doi : 10.1002/pssb. 2221330150.

8. Saichev A. I., Zaslavsky G. M. Fractional kinetic equations: solutions and applications, Chaos, 1997, vol. 7, no. 4, pp. 753-764. doi: 10.1063/1.166272.

9. Repin O. A., Kumykova S. K. A problem with generalized fractional integro-differentiation operators of arbitrary order, Russian Math. (Iz. VUZ), 2012, vol.56, no. 12, pp. 50-60. doi : $10.3103 / \mathrm{S} 1066369 \times 12120067$.

10. Bitsadze A. V. Theory of equations of mixed type, whose order degenerates along the line of change of type, Mekhanika sploshnoi sredy $i$ rodstvennye problemy analiza [Continuous Medium Mechanics and Related Problems of Analysis]. Moscow, Nauka, 1972, pp. 47-52 (In Russian).

11. Samko S. G., Kilbas A. A., Marichev O. I. Integraly $i$ proizvodnye drobnogo poriadka $i$ nekotorye ikh prilozheniia [Integrals and derivatives of fractional order and some of their applications]. Minsk, Nauka i tekhnika, 1987, 688 pp. (In Russian)

12. Kumykova S. K. A Problem with Nonlocal Boundary Conditions on the Characteristics for an Equation of Mixed Type, Differ. Uravn., 1974, vol. 10, no. 1, pp. 78-88 (In Russian).

13. Tricomi F. G. Integral equations, Pure and Applied Mathematics, vol.5. New York, Interscience Publ., Inc, 1957, viii+238 pp.

Received 23/X/2014;

received in revised form $05 / \mathrm{XI} / 2014$;

accepted $27 / \mathrm{XI} / 2014$. 